University of Nebraska - Lincoln

DigitalCommons@University of Nebraska - Lincoln

U.S. Department of Veterans Affairs Staff

Publications

U.S. Department of Veterans Affairs

1999

\title{
Mood state and recent cocaine use are not associated with levels of cocaine cue reactivity
}

\author{
Steven J. Robbins \\ University of Pennsy/vania, robbins@beaver.edu \\ Ronald N. Ehrman \\ University of Pennsylvania \\ Anna Rose Childress \\ University of Pennsylvania, childres@mail.med.upenn.edu \\ James Cornish \\ University of Pennsylvania, CORNISH@RESEARCH.TRC.UPENN.EDU \\ Charles P. O’Brien \\ University of Pennsy/vania, obrien@upenn.edu
}

Follow this and additional works at: https://digitalcommons.unl.edu/veterans

Robbins, Steven J.; Ehrman, Ronald N.; Childress, Anna Rose; Cornish, James; and O'Brien, Charles P., "Mood state and recent cocaine use are not associated with levels of cocaine cue reactivity" (1999). U.S. Department of Veterans Affairs Staff Publications. 67.

https://digitalcommons.unl.edu/veterans/67

This Article is brought to you for free and open access by the U.S. Department of Veterans Affairs at DigitalCommons@University of Nebraska - Lincoln. It has been accepted for inclusion in U.S. Department of Veterans Affairs Staff Publications by an authorized administrator of DigitalCommons@University of Nebraska - Lincoln. 


\title{
Mood state and recent cocaine use are not associated with levels of cocaine cue reactivity
}

\author{
Steven J. Robbins ${ }^{\mathrm{a}, \mathrm{b}, \mathrm{c}, *}$, Ronald N. Ehrman ${ }^{\mathrm{a}, \mathrm{b}}$, Anna Rose Childress ${ }^{\mathrm{a}, \mathrm{b}}$, \\ James W. Cornish ${ }^{\mathrm{a}, \mathrm{b}}$, Charles P. O’Brien ${ }^{\mathrm{a}, \mathrm{b}}$ \\ ${ }^{a}$ Treatment Research Center, University of Pennsylvania, 3900 Chestnut Street, Philadelphia, PA 19104, USA \\ b Veterans Affairs Medical Center, Philadelphia, PA 19104, USA \\ ${ }^{\mathrm{c}}$ Department of Psychology, Beaver College, 450 South Easton Road, Glenside, PA 19038, USA
}

Received 29 July 1999; accepted 11 August 1999

\begin{abstract}
Eighty-one cocaine-dependent outpatients were assessed for their reactions to cocaine-related cues in a laboratory setting. All subjects contributed a urine sample prior to the session. Compared with non-drug control cues, the cocaine stimuli produced increases in physiological arousal, self-reports of high, craving, and withdrawal, and self-reports of negative mood. Subjects who tested cocaine-positive on the day of testing differed only in skin resistance responding from those who tested cocaine-negative. Changes in cue-induced physiological and self-report measures were also not associated with between-subject variations in mood as measured by the Profile of Mood States (POMS) questionnaire administered prior to cue assessment. Thus, variations in baseline mood and recent cocaine use history do not introduce an additional source of variability in cue reactivity measurements. However, negative mood states at the start of a session were associated with higher levels of self-reported craving, high, and withdrawal both before and after cue exposure. (C) 2000 Elsevier Science Ireland Ltd. All rights reserved.
\end{abstract}

Keywords: Cue reactivity; Cocaine dependence; Mood state; Craving; Cocaine use

\section{Introduction}

Many studies have demonstrated that cocaine-dependent individuals show alterations in mood, increases in craving, and increases in physiological arousal when presented with cocaine cues in the laboratory (Childress et al., 1988; Ehrman et al., 1992; Berger et al., 1996; Robbins et al., 1997; Reid et al., 1998). Such reactivity is thought to provide some of the motivation to relapse to cocaine use following treatment. As a result, many laboratories have begun using measures of cue reactivity as outcome measures in trials of anti-craving medications (e.g. Kranzler and Bauer, 1992; Robbins et al., 1992; Alim et al., 1994; Rosse, et al., 1994; Alim et al., 1995; Satel et al., 1995; Berger, et al., 1996; Ehrman et al., 1996). Such measurements are thought to have at

\footnotetext{
* Corresponding author. Present address: Department of Psychology, Beaver College, 450 S. Easton Road, Glenside, PA 19038, USA. Tel.: + 1-215-5722987.

E-mail address: robbins@beaver.edu (S.J. Robbins)
}

least two advantages over traditional retrospective reports of cocaine craving.

First, retrospective reports require subjects to accurately estimate a subjective state that occurred hours or days before the time of measurement. Such reports obviously require that subjects be accurate and reasonably precise at recalling intensities of past internal states. Unfortunately, two decades of memory research has documented that recall, and particularly recall of quantitative information, is often subject to reconstruction (e.g. Loftus, 1975) and to distortion by cognitive shortcuts or heuristics (e.g. Tversky and Kahneman, 1973, 1974). Because cue reactivity studies require subjects to report on a state of craving as they currently experience it, such biases are largely eliminated.

Second, retrospective craving reports may be influenced by idiosyncratic events or experiences occurring during the days upon which the report is based. Subject differences in history could include differential exposure to real world drug cues, availability of money to buy drugs, or various mood-altering experiences. Between 
subject differences in such personal experiences may obscure the effects of a subsequent treatment by introducing an uncontrolled source of within-group variability in the measurement of craving. Cue reactivity studies reduce this problem by exposing all subjects to a common set of drug-related events. This technique not only reduces between-subject variance by examining craving under constant conditions, but also measures craving under the high-risk environment conditions of most clinical concern.

Cue exposure measurements are not without disadvantages, however. The lack of drug availability in a laboratory session may reduce craving levels, as might the inability to adequately reproduce the full range of real-world drug events. Furthermore, despite exposure to a standardized set of laboratory cues, subjects may be influenced by extra-session variables. In particular, recent cocaine use and negative mood states at the time of testing are two factors which have been shown previously to influence self-reported craving. We will review each of these factors in turn.

A number of studies have documented increases in craving immediately following the administration of cocaine in a laboratory setting (Jaffe et al., 1989; Fischman et al., 1990; Foltin and Fischman, 1997). Consequently, recent cocaine use may well lead to increases in cue-induced craving as well. Cocaine use could affect cue reactivity in other ways as well. If the patient is under pressure from a treatment program to achieve abstinence at the time of cue screening, then a recent lapse could be especially distressing and lead to enhanced levels of cue-invoked anxiety or stress. Alternatively, recent drug use could reduce levels of cue responding by satisfying an individual's craving for the drug. In any case, differences in recent drug use between individuals could confound the measurement of treatment effects on cue reactivity.

Cue reactivity studies employing inpatients avoid the potentially confounding influence of recent cocaine use. However, studies often involve outpatient subjects who continue to use cocaine throughout the trial. For example, Ehrman et al. (1998) found that 17 of $69(25 \%)$ outpatients enrolled in a study of ritanserin provided cocaine-positive urine samples on the day of the laboratory cue session. Therefore, the influence of cocaine use on reactivity represents a real concern in such studies.

Variations in general mood states may also serve to modulate craving to laboratory cocaine cues. For example, Baker et al. (1987) proposed that craving may result from either negative affect (withdrawal-based urges) or from positive affect (incentive-based or appetitive urges). Many studies employing correlational designs have now documented that fluctuations in mood states often covary with fluctuations in craving (e.g. Sherman et al., 1986; Weddington et al., 1990; Satel et al., 1991; Kowatch et al., 1992; Tunis et al., 1994; Covi et al., 1995). Because correlational studies cannot be used to draw the conclusion that negative mood states cause craving, many investigators have used hypnosis or short scripts to experimentally induce mood states. For example, Tiffany and colleagues have shown that short scripts with emotionally negative content can produce craving in cigarette smokers (e.g. Tiffany and Drobes, 1990; Maude-Griffin and Tiffany, 1996). Unfortunately, other studies have failed to confirm these results (e.g. Drobes et al., 1994; Elash et al., 1995; Droungas et al., 1995). Induced negative moods have also produced craving in individuals who abuse heroin and alcohol (Sherman et al., 1989; Litt et al., 1990; Childress et al., 1994; Cooney et al., 1997).

The production of craving by experimentally-produced negative moods suggests that mood states may influence the outcome of cue reactivity studies. Consequently, a number of studies have examined the joint influence of drug cues and mood states on craving. Unfortunately, the results of such studies have been mixed (see Cooney et al., 1997 for a review). A number of reports have found that negative moods enhance cue-induced craving states (e.g. Greeley et al., 1992; Elash et al., 1995; Maude-Griffin and Tiffany, 1996; Cooney et al., 1997). However, other studies have failed to obtain such effects (Litt et al., 1990; Tiffany and Drobes, 1990; Childress et al., 1994; Shadel et al., 1998). To our knowledge, no studies have yet examined the influence of mood states on cocaine cue reactivity. Given the widespread use of cue reactivity as a measurement tool in cocaine treatment studies, such investigations seem warranted.

In order to examine the impact of recent cocaine use and mood on cue reactivity, we examined laboratory responding in a sample of outpatient subjects who were enrolled in a medication trial. All subjects were exposed to the cues during a baseline session prior to the start of medication. Subjects provided a urine sample on the day of testing and completed the Profile of Mood States questionnaire (POMS; McNair et al., 1971) prior to cue exposure. Because subjects were participating in a larger medication protocol, experimental manipulations of mood state were not possible. Consequently, this study employed a correlational design in which subject variations in mood state and cocaine use prior to the cocaine cue session were used as predictor variables and cue-induced changes in physiological responding, selfreported moods, and self-reported drug states were used as dependent measures.

The purpose of these analyses was to determine whether levels of cue reactivity are associated with baseline differences in mood state or drug use. If so, then future studies would need to control for such effects either by explicit experimental manipulations (e.g. by employing inpatient rather than outpatient 
subjects or by inducing mood states) or by using covariance analyses incorporating the confounding factors. The present study was also intended to address clinical concerns that subjects who have recently used cocaine or are in negative mood states might be at risk for excessively high (clinically problematic) levels of craving in response to cocaine cues. If such a finding were obtained, then additional exclusion criteria might be required in future cue reactivity studies or in clinical applications of cue exposure.

\section{Methods}

\subsection{Research participants}

The participants were 81 males who met DSM-III-R criteria for cocaine dependence and were enrolled in outpatient treatment at a Veterans Affairs Medical Center. All subjects gave informed, voluntary consent to participate in cocaine cue reactivity sessions. Subjects also gave consent to participate in a medication study of ritanserin, a 5-HT2 receptor antagonist (see Ehrman et al., 1996). Demographic information on the subjects was collected through use of an interview including the Addiction Severity Index (ASI; McLellan et al., 1980) conducted during treatment intake. Seventy-eight subjects were black, one was white, and two were Hispanic. Subjects' average age was $37.9($ S.D. $=6.1)$ and they had completed an average of $12.6($ S.D. $=1.4)$ years of education. Subjects reported having used cocaine for 10.7 (S.D. $=8.3$ ) of the 30 days prior to the interview, spending an average of US\$ 544 (S.D. $=631$ ) for drugs during that month. They reported having used cocaine regularly for an average of 6.3 (S.D. $=4.6)$ years prior to treatment admission. Seventy-five subjects reported that their usual route of administration was by smoking crack cocaine; six subjects injected the drug intravenously. Reactivity data from a subset of these subjects were reported on in a previous paper describing the effects of the medication ritanserin on cue reactivity (Ehrman et al., 1996).

\subsection{Procedures}

\subsubsection{Cue session procedures}

All subjects participated in a cocaine cue session and in two control cue sessions prior to the start of the medication study. The sessions took place on separate days within the same week. Time of day of the sessions was not explicitly controlled but the order of session presentations was balanced across subjects. One of the control sessions consisted of a pilot test of cues intended to be physiologically arousing but non-drug related. Data from that session are not included here. The present paper presents findings from the cocaine cue session and from the control session employing a standard set of non-drug control cues.

Upon arrival at the laboratory for the cue session, participants were administered the POMS questionnaire which requires rating the intensity of 65 mood-related adjectives on a $0-4$ scale. Item ratings are then combined into six summary scales or factor scores labeled vigor, depression, tension, anger, fatigue, and confusion. The POMS has been used extensively to document both the direct effects of cocaine administration (e.g. Fischman et al., 1983a,b, 1985) and the effects of cocaine abstinence (e.g. Weddington et al., 1990; Kowatch et al., 1992; Rhoades et al., 1993; Tunis et al., 1994; Covi et al., 1995; van Gorp et al., 1999). Subjects were then asked to sit down in the sound-attenuated recording chamber where surface recording electrodes were attached and interfaced with a polygraph in an adjacent control room.

Following electrode placement, subjects were asked to rate the intensity of any feelings of cocaine-related high, withdrawal, or craving. Answers were recorded on a $0-10$ scale with anchor points provided at 0 ('not at all'), 3 ('mildly'), 7 ('moderately') and 10 ('extremely'). Following these self-reports, physiological recording was begun. During the initial baseline period of $15 \mathrm{~min}$, subjects sat quietly and no programmed events were provided. Next, the subjects listened to an audiotape for $10 \mathrm{~min}$, watched a videotape for $10 \mathrm{~min}$, and performed a manual task for 5 min. Two-minute rest intervals separated presentations of these events. Following completion of physiological recording, subjects were again asked to rate their levels of high, withdrawal, and craving and were readministered the POMS. At the end of the cocaine cue session, subjects were seen by an experienced therapist to discuss any craving that may have persisted beyond cue presentations. Subjects met with the therapist until craving levels had declined to pre-session levels. Counseling sessions typically lasted no more than $15-20 \mathrm{~min}$.

\subsubsection{Content of the cocaine and non-drug cues}

Stimuli presented during the cocaine cue session were matched to each subject's typical route of cocaine administration. Subjects who injected cocaine intravenously listened to an audiotape of patients discussing cocaine injections, watched a videotaped simulation of the preparation and insertion of a syringe, and prepared a syringe in the usual way during the task. Syringe preparation involved the use of a lactose solution.

For subjects who smoked cocaine, the audiotape consisted of a discussion of the effects of smoking cocaine. Two videotapes were available for these subjects. Patients who prepared their own cocaine crystals ('freebasers') watched a video which depicted two individuals making freebase cocaine and then pretending to 
smoke the precipitate. Patients who bought already packaged crystal cocaine (crack) watched a videotape in which two individuals opened vials containing simulated crack cocaine and pretended to smoke the crystals. During the task, patients either performed a simulated freebasing ritual involving benzocaine in the place of cocaine, or handled crack vials filled with crystallized benzocaine.

All subjects were exposed to the same set of stimuli during the control session. The audiotape consisted of a Wang word processing instructional tape, the video consisted of an excerpt from a nature video, and the task consisted of sorting a deck of Old Maid cards into matching pairs.

\subsubsection{Physiological recording procedures}

Physiological recording was accomplished through the use of $\mathrm{Ag} / \mathrm{AgCl}$ surface recording electrodes interfaced with a Grass model 7E polygraph located in a separate control room. Two heart rate electrodes were employed, one under the left clavicle and one under the lowest rib on the subject's left side. Skin resistance electrodes were placed on the second phalanx of the index and middle fingers of the non-dominant hand. The first phalanx of the ring finger of the non-dominant hand served as the site for the skin temperature electrode. Analog data from the polygraph were amplified, converted to digital format, and stored on a computer for later analysis (see Robbins et al., 1999 for more details and equipment specifications).

\subsubsection{Collection and analysis of urine samples}

All subjects submitted a urine sample just prior to each session. Analysis of the samples was performed using the fluorescence polarization immunoassay technique, which measures levels of the cocaine metabolite benzoylecgonine. Values greater than $300 \mathrm{ng} / \mathrm{ml}$ were classified as cocaine positive. Past work has demonstrated that benzoylecgonine levels frequently remain detectable for three or more days following cocaine use (Hawks and Chiang, 1986; Burke et al., 1990). Therefore, although a positive test value indicates that cocaine use occurred at some point in the few days prior to cue presentation, such a value does not necessarily mean that the subject was under the effects of cocaine at the time of the session. Urine samples were also analyzed for the presence of amphetamines, benzodiazepines, barbiturates, opiates, cannabis, and methadone.

\section{Results}

\subsection{General analytic plan}

Three sets of analyses are presented below. First, we compared responding between cocaine and non-drug cues across all subjects. Those analyses involved two-way repeated measures ANOVAs with session as one withinfactor and time (pre-post) as the second within-factor. Evidence for response specificity to the cocaine cues would come from session by time interactions in each analysis. Levels of reactivity within each session were then examined through paired $t$-tests comparing pre- and post-cue responding. All these analyses employed a significance threshold of $\alpha=0.05$.

The next two sets of analyses looked at the association of recent drug use status and mood state with measures of cue reactivity. Because the purpose of these analyses was to establish whether reactions to cocaine cues are modulated by mood state or drug use, only data from the cocaine cue session were included.

The second set of analyses involve splitting subjects into two groups: those who tested cocaine-positive prior to the cocaine cue session and those who tested cocainenegative. Differences in reactivity between these groups were analyzed by means of two-way ANOVAs in which the group served as a between-factor and time (pre-post) served as a within-factor. Changes in physiology, POMS scores, and self-reported drug states during the cocaine cue session served as dependent measures. An association between prior cocaine use and levels of cue reactivity would be revealed as a group by time interaction in each analysis. Once again, all tests utilized a significance threshold of $\alpha=0.05$ in order to maximize sensitivity to possible group differences.

Finally, we examined whether cue reactivity was associated with variations in baseline mood. For these analyses, we first calculated Pearson $r$ correlations between each of the six POMS subscale scores collected at baseline and cocaine cue-induced changes in physiology and self-reported drug states (high, withdrawal, craving). If more than one POMS score was significantly $(P<$ $0.05)$ correlated with a given set of change scores, then simultaneous multiple regression analyses were performed.

In all the analyses, physiological responding to the cues was summarized as mean responding during the last 5 min of the video stimuli. Cue reactivity studies in our laboratory have repeatedly obtained evidence that physiological arousal during drug cue sessions increases across stimulus presentations, presumably because subjects become increasingly involved in imagining real drug situations. For this reason, we have typically summarized cue responding as mean responding during the last 10 min of the session (last $5 \mathrm{~min}$ of the video and last $5 \mathrm{~min}$ of the task). In the present study, the card-sorting task used in the non-drug session appeared to produce greater motion artifact problems than seen with previous control events. Consequently, physiological responding in this study was summarized as mean responding over the last 5 min of the videotape. This period represented the last portion of the session in which all subjects were exposed to cocaine or control stimuli while completely at rest. 
Table 1

Physiological responding at baseline and the change produced by video presentations during the cocaine and non-drug cue sessions ${ }^{\text {a }}$

\begin{tabular}{lccc}
\hline Measure & $N$ & Cocaine cue session & Non-drug cue session \\
\hline \multicolumn{2}{l}{ Skin temperature $\left({ }^{\circ} \mathrm{F}\right)$} & & \\
Baseline & 77 & $93.1(4.8)$ & $91.8(6.2)$ \\
Video change & 77 & $-4.4(3.6)$ & $+0.1(3.5)$ \\
Skin resistance & $(k \Omega)$ & & $318.0(184.6)$ \\
Baseline & 73 & $333.2(199.3)$ & $+28.0(56.5)$ \\
Video change & 73 & $-54.0(94.2)$ & \\
Heart rate (beats $/ \mathrm{min})$ & & $72.0(9.0)$ \\
Baseline & 78 & $72.3(9.3)$ & $-2.0(4.8)$ \\
Video change 78 & $+1.6(4.9)$ &
\end{tabular}

${ }^{a}$ Baseline physiology represents mean responding during the last 5 min of the baseline period. Video change scores were calculated by subtracting the baseline value from mean responding during the last 5 min of the video. All values are means (S.D.). Sample sizes vary due to missing data.

\subsection{Comparing reactivity to cocaine and non-drug cues}

\subsubsection{Physiological responding}

Reactions to the cues were summarized by subtracting mean responding during the last $5 \mathrm{~min}$ of the baseline period from mean responding during the last $5 \mathrm{~min}$ of the video stimulus. Results for the two sessions are depicted in Table 1. In order to compare responding between the sessions, each physiological measure was analyzed using a two-way repeated measures ANOVA with session (cocaine cue vs. non-drug cue) as one within-factor and time (baseline vs. video) as the second within-factor. A separate analysis was run for each of the three physiological measures. Each of the three session by time interactions was significant (heart rate: $F(1,77)=24.85, \quad P<0.001 ; \quad$ skin temperature: $F(1,76)=63.80, P<0.001$; skin resistance: $F(1,72)=$ 49.70, $P<0.001)$.

Inspection of Table 1 reveals that the two sessions had opposite effects on each of the physiological measures.
Cue-induced changes were examined using paired $t$-tests comparing response during the video and baseline periods. The cocaine video caused significant decreases in skin temperature $(t(76)=10.76, P<0.001)$ and skin resistance $(t(72)=4.90, P<0.001)$ and a significant increase in heart rate $(t(77)=2.90, P<0.01)$. By contrast, the non-drug cues caused heart rate to decrease $(t(77)=3.80, P<0.001)$ and skin resistance to increase $(t(72)=4.23, P<0.001)$. Skin temperature was not affected by the control cues. Thus, the cocaine cues caused significant and specific increases in physiological arousal across all three measures.

\subsubsection{Self-reported mood states}

Mood responding was summarized as the mean group score for each of the six summary scales on the POMS. Scores obtained before and after each session are shown in Table 2. In order to compare reactivity during the two sessions, the data were analyzed using a two-way repeated measures ANOVA with the session (cocaine or non-drug) as one within-factor and time (pre- or postcues) as the other within-factor. Significant session by time interactions were revealed for every POMS scale except fatigue (anger: $F(1,75)=8.75, P<0.005$; confusion: $F(1,74)=10.21, P<0.005$; depression: $F(1,73)=$ 10.53, $P<0.005$; tension: $F(1,71)=27.49, P<0.001$; vigor: $F(1,72)=4.15, P<0.05)$. Inspection of the means in Table 2 reveals that these interactions all resulted from the cocaine cues causing increases in negative mood states, while the non-drug cues caused decreases or no change at all.

Planned comparisons using paired $t$-tests were performed to compare post-cue with pre-cue response in each session. As summarized in Table 2, the cocaine cues produced significant increases in anger $(t(75)=2.21$, $P<0.05)$, confusion $(t(74)=3.23, P<0.01)$, depression $(t(73)=2.80, P<0.01)$, and tension $(t(71)=4.58, P<$ $0.001)$ and a significant decrease in vigor $(t(72)=3.36$, $P<0.005)$. The non-drug cues actually produced minor

Table 2

Mean self-report scores on mood and drug state before and after exposure to the cocaine and non-drug cues ${ }^{\text {a }}$

\begin{tabular}{|c|c|c|c|c|c|c|c|}
\hline & $N$ & Cocaine pre-cue & Cocaine post-cue & $P$ & Non-drug pre-cue & Non-drug post-cue & $P$ \\
\hline \multicolumn{8}{|l|}{ POMS } \\
\hline Anger & 76 & $5.0(7.7)$ & $6.2(9.0)$ & 0.03 & $3.9(6.2)$ & $3.1(6.3)$ & 0.03 \\
\hline Confusion & 75 & $4.4(3.4)$ & $5.4(4.7)$ & 0.002 & $4.4(3.7)$ & $4.2(3.5)$ & NS \\
\hline Depression & 74 & $8.8(8.5)$ & $10.4(10.2)$ & 0.007 & $7.2(8.5)$ & $6.6(8.4)$ & NS \\
\hline Fatigue & 76 & $3.1(3.9)$ & $3.3(4.6)$ & NS & $3.0(3.9)$ & $3.5(4.4)$ & NS \\
\hline Tension & 72 & $5.9(4.5)$ & $9.4(7.3)$ & 0.001 & $5.5(4.1)$ & $4.6(3.4)$ & 0.002 \\
\hline Vigor & 73 & $17.7(7.2)$ & $16.1(8.1)$ & 0.001 & $17.3(6.9)$ & $16.9(7.0)$ & NS \\
\hline \multicolumn{8}{|l|}{ Drug state } \\
\hline Craving & 78 & $2.1(2.4)$ & $4.6(3.3)$ & 0.001 & $1.9(2.2)$ & $0.9(1.6)$ & 0.001 \\
\hline High & 79 & $0.4(1.2)$ & $2.1(2.8)$ & 0.001 & $0.3(1.1)$ & $0.3(1.1)$ & NS \\
\hline Withdrawal & 78 & $1.0(1.8)$ & $2.0(2.6)$ & 0.001 & $0.9(1.9)$ & $0.6(1.6)$ & 0.054 \\
\hline
\end{tabular}

${ }^{a}$ All values are means (S.D.). Sample sizes vary due to missing data. $P$-values represent the results of paired $t$-tests comparing pre- and post-cue means for each session. NS refers to $P$-values $>0.05$. 
changes in the opposite direction. Subjects showed significant decreases in anger $(t(75)=2.23, P<0.05)$ and tension $(t(71)=3.20, P<0.005)$ to the non-drug cues. None of the other four scales showed significant changes in response to the control stimuli. In sum, the cocaine cues produced highly specific increases in negative mood compared to control stimuli.

\subsubsection{Self-reported drug states}

Mean reported levels of craving, high, and withdrawal before and after exposure to the two sets of cues are also depicted in Table 2 . Once again, differences in reactivity to the two sets of cues were examined by means of a two-way repeated measures ANOVA using session and time as the two within-factors. Significant interactions were revealed for all three drug self-reports (craving: $F(1,77)=87.33, P<0.001$; high: $F(1,78)=31.82, P<$ 0.001 ; withdrawal: $F(1,77)=14.94, P<0.001)$. Paired $t$-tests comparing pre- and post-cue responding found that the cocaine cues caused significant increases in craving $(t(77)=8.33, P<0.001)$, high $(t(78)=6.01, P<$ $0.001)$, and withdrawal $(t(77)=3.69, P<0.001)$. The non-drug cues caused a significant decrease in craving $(t(77)=4.84, P<0.001)$, a decrease in withdrawal that just missed the significance threshold $(t(77)=1.96, P=$ .054 ), and no change in high.

\subsection{Association of baseline cocaine use with levels of cue reactivity}

Subjects were divided into two groups based on the results of the urine test given on the day of the cocaine cue session. Fifty subjects tested negative for cocaine (group NEG) and 31 tested positive (group POS). Four subjects in each of these groups also tested positive for cannabis. In group NEG, one subject tested positive for barbiturates and one tested positive for benzodiazepines. No subjects in either group submitted urine samples which revealed the use of amphetamines, opiates, or methadone.

\subsubsection{Physiological responding}

Group differences in responding to the cocaine video were examined by means of a two-way ANOVA with group (POS or NEG) as the between factor and session period (baseline vs. video) as the within factor. A separate analysis was run for each of the three physiological measures. The group by session interaction was significant only for the analysis of skin resistance; subjects in group POS showed a greater drop in skin resistance than subjects in group NEG $(F(1,74)=5.96, P<0.05)$. In group POS, mean skin resistance dropped from $469.3 \mathrm{k} \Omega$ (S.D. $=211.5)$ during baseline to $381.5 \mathrm{k} \Omega$ (S.D. $=189.9)$ during the video. For group NEG, the baseline and video scores were 261.8 (S.D. $=144.2$ ) and 226.5 (S.D. $=$ 140.2), respectively. Paired $t$-tests revealed that both of these declines were statistically significant (group POS: $t(26)=5.00, P<0.001$; group NEG: $t(48)=2.79, P<$ $0.01)$.

\subsubsection{Self-reported mood states}

Between-group differences in POMS scores were examined using two-way, repeated measures ANOVAs with group (POS or NEG) as the between factor and time (pre- or post-session) as the within factor. Separate analyses were performed for each of the six POMS subscales. As described earlier, the cocaine cues generally caused increases in negative mood states (see Table 2). However, none of the group by time interactions were significant, demonstrating that none of these changes in negative mood differed across the groups.

\subsubsection{Self-reported drug states}

As described earlier, subjects showed significant increases in high, withdrawal, and craving in response to the cocaine cues (see Table 2). However, two-way, repeated measures ANOVAs revealed no group by time interactions. Thus, increases in self-reported drug states did not differ between subjects who tested positive or negative for cocaine at the time of cue exposure.

\subsection{Association of baseline mood with levels of cue reactivity}

In order to examine the association between baseline mood and cue reactivity, we first calculated Pearson $r$ correlations between each of the six POMS scores and the physiological and drug state change scores produced in response to the cocaine cues. Thus, there were a total of 36 correlations (six POMS scores $\times$ six dependent measures). Of these correlations, only three reached statistical significance. Cue-induced changes in self-reported craving were significantly correlated with pre-cue levels of vigor $(r=-0.23, P=0.043)$, depression $(r=$ $0.23, P=0.043)$, and fatigue $(r=0.27, P=0.016)$. If a more conservative alpha value of 0.01 or below is used to correct for the multiple tests, then none of the correlations would have reached significance.

In order to explore the correlations with craving further, we performed a simultaneous multiple regression analysis using vigor, depression, and fatigue scores as the predictor variables and craving change score as the dependent variable. The resulting model produced an overall $F$ statistic of $2.62(P=0.057)$, a multiple $r$ value of 0.312 , and an $r^{2}$ value of 0.097 . None of the three predictor variables contributed a statistically significant amount of variance to the model (all $P$ values $>0.20$ ).

Finally, we calculated Pearson $r$ correlations between scores on each of the baseline POMS subscales and reports of high, withdrawal, and craving before and after the session. Those correlations are shown in Table 3. As seen in the table, 30 of the 36 correlations are significant 
Table 3

Drug and Alcohol Dependence 59 (2000) 33-42

Correlations between scores on each of the precue POMS subscales and reports of high, withdrawal, and craving before and after cue exposure ${ }^{\text {a }}$

\begin{tabular}{|c|c|c|c|c|c|c|}
\hline & \multicolumn{2}{|l|}{ Craving } & \multicolumn{2}{|l|}{ High } & \multicolumn{2}{|c|}{ Withdrawal } \\
\hline & Pre-cue & Post-cue & Pre-cue & Post-cue & Pre-cue & Post-cue \\
\hline Anger & $0.33 * *$ & $0.25 *$ & $0.29 *$ & 0.12 & $0.33 * *$ & 0.11 \\
\hline Confusion & $0.29 * *$ & $0.32 * *$ & $0.44 * *$ & $0.34 * *$ & $0.33 * *$ & $0.26 *$ \\
\hline Depression & $0.41 * *$ & $0.44 * *$ & $0.43 * *$ & $0.27 *$ & $0.40 * *$ & $0.29 *$ \\
\hline Fatigue & $0.23 *$ & $0.36^{* *}$ & $0.38 * *$ & 0.21 & $0.23 *$ & $0.24 *$ \\
\hline Tension & $0.33 * *$ & $0.28 *$ & $0.27 *$ & $0.29 *$ & $0.36 * *$ & $0.26 *$ \\
\hline Vigor & $-0.28 *$ & $-0.31 * *$ & -0.20 & -0.12 & $-0.23 *$ & -0.17 \\
\hline
\end{tabular}

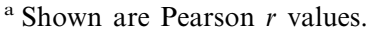

$* P<0.05$.

** $P<0.01$

at the 0.05 level. The five negative mood states (anger, confusion, depression, fatigue, and tension) were positively correlated with all three drug states while the vigor subscale showed negative correlations with the drug-related self-reports. Thus, subjects who reported higher levels of craving, high, and withdrawal both before and after the cue session also reported more negative mood states before the session.

\section{Discussion}

Subjects in this study showed significant increases in arousal, self-reported drug states, and self-reported negative mood following exposure to laboratory cocaine cues. The cocaine cue responses were similar in form and magnitude to those recorded in past studies in our laboratory (e.g. Ehrman et al., 1992; Robbins et al., 1992, 1999) and differed in both form and magnitude from reactivity produced by non-drug control events. However, variations in reactivity to the cocaine cues were generally not associated with between-subject differences in recent cocaine use and mood state prior to the session.

Subjects who provided a cocaine-positive urine sample on the day of cue assessment did not differ on 11 of 12 cue reactivity measures from subjects who tested cocainenegative. The two groups did not show different cue-elicited changes in any of the six POMS scores, in reports of high, withdrawal, and craving, or in heart rate and skin temperature change scores. Subjects in group POS did show a greater drop in skin resistance than subjects in group NEG. However, subjects in group POS also had baseline skin resistance scores that were nearly double those of group NEG. Thus, it is unclear whether this single difference between groups reflects a true association between cocaine use and cue reactivity, an artifact of a highly elevated baseline, or an instance of type 1 error. In any case, the negative results on 11 of 12 measures suggest that cocaine use prior to a cue reactivity session has at best only a minimal relationship to cue-elicited responding.
The present study also failed to uncover significant associations between baseline POMS scores and cue reactivity. Of 36 correlations between the six POMS scores and the six cue reactivity measures, only three were significant at the 0.05 level (vigor, depression, and fatigue were correlated with cue-elicited craving). Given the 0.05 significance level used, two of the correlations would be expected to reach significance by type 1 error alone. Had a more conservative 0.01 significance level been employed, none of the correlations would have reached significance. Furthermore, a multiple regression analysis using the vigor, fatigue, and depression scores as predictors failed to account for a significant proportion of the variance in craving.

The present results indicate that laboratory measures of cocaine cue reactivity are not significantly associated with temporally contiguous external events such as normal variations in subject mood or recent cocaine use. Thus, laboratory measures of cue-induced craving serve to eliminate many sources of uncontrolled variance which otherwise might influence subject self-reports. By reducing sources of variability across subjects within a commonly treated group, cue exposure sessions provide a more powerful assessment of potential differences between groups in treatment studies.

Several limitations of the present data are important to note. First, although the presence of a cocaine-positive urine sample on the day of cue assessment demon-strates that a subject has used cocaine sometime in the few days prior to testing, the urine result cannot be used to determine whether the subject is actually experiencing drug effects (such as intoxication or withdrawal) at the time of testing. Thus, the present results do not rule out the possibility that drug use occurring immediately before testing may affect a subject's responses. In our studies, laboratory technicians are instructed to exclude subjects from testing if they appear to be under the influence of drugs at the start of a session. Thus, it is unlikely that any of the subjects were actually in a drug-induced state at the time of testing (in fact, no 
participants appeared to be intoxicated at the time of testing). As noted earlier, previous laboratory studies have shown that craving does increase immediately following cocaine use (Jaffe et al., 1989; Fischman et al., 1990; Foltin and Fischman, 1997). Thus, we would continue to recommend excluding subjects from cue exposure who appear to be under the influence of drugs at the time of testing.

A second limitation concerns the range of mood states reported by patients on the POMS questionnaire. In general, the mean subscale scores recorded here were similar to those reported in other treatment studies of cocaine-dependent individuals (e.g. Weddington et al., 1990; Covi et al., 1995; van Gorp et al., 1999) but much less extreme than those produced by psychiatrically-impaired groups of individuals in the original validation studies of the POMS questionnaire (McNair et al., 1971). Thus, the present data do not address whether more extreme mood scores might influence cue responding. Furthermore, because the present study employed only a single cue reactivity session, the results cannot be used to conclude that within-subject variations in mood state are unrelated to cue-induced craving and physiological arousal. Different individuals may be particularly susceptible to cocaine cues in the presence of different mood states. In that case, variations in a particular mood state may lead to variations in cue responding for some subjects but not for others. Although the present study demonstrates that betweensubject variations in mood are unrelated to cue responding, these results do not address the effects of within-subject variations.

Although the limited range of baseline moods measured in this study place limits on its generalizability to more psychiatrically-impaired populations, it is important to remember that the individuals studied here represent a typical group of cocaine abuse patients seen in our outpatient cocaine treatment center. Thus, the results do demonstrate that the typical range of moods displayed by our usual sample of largely male, African-American, lower SES patients are not associated with variations in cue reactivity. The external validity of this finding should be no less than the external validity of any other results produced with such a sample. Furthermore, the average POMS scores recorded in this study were quite similar to those recorded in earlier studies with cocaine-dependent patients (e.g. Weddington et al., 1990; Covi et al., 1995; van Gorp et al., 1999). Consequently, there is little reason to believe that the range of POMS scores recorded in this study were substantially lower than those typically obtained from cocaine-dependent outpatients.

A third limitation in this study concerns the use of single-item scales to measure high, withdrawal, and craving. Some authors have argued that single-item scales are inadequate to capture the multifaceted nature of subjective states such as craving (e.g. Tiffany et al., 1993). As an alternative, Tiffany et al. (1993) proposed that craving studies utilize a 45 -item cocaine craving questionaire (CCQ) which identifies separate dimensions of the craving state. Although the three drug state questions used here do not capture the multidimensional nature of subjective drug effects, they do have several virtues in their favor. First, cocaine dependent patients provide significantly different scores on these items when faced with cocaine versus non-cocaine cues (Ehrman et al., 1992; Table 2 of the present paper). Second, non-drug users provide lower reports on these scales in response to cocaine cues than do cocaine dependent outpatients (Ehrman et al., 1992). Finally, previous work in our laboratory has shown that an increase across single-item craving scores collected three days apart predicts an increased likelihood of cocaine use during that 3-day period (Robbins and Ehrman, 1998). To our knowledge, the CCQ has not been similarly validated against the results of repeated urine tests.

A final limitation of these results involves the lack of information on pre-session caffeine or nicotine use. In principle, variations across participants in use of these stimulants could produce an additional source of variability in baseline physiology or in physiological responses to the cues. Such variability could have reduced the power of the present analyses to detect relationships between pre-cue variables and cue reactivity. Some evidence for the interaction of nicotine use with cocaine cue reactivity comes from a recent study by Reid et al. (1998). They found that pre-cue nicotine use enhanced craving produced by the cocaine cues but had no effect on skin temperature or skin conductance responding. This effect of nicotine on craving suggests that future studies further explore the potential impact of nicotine and caffeine on cocaine cue reactivity.

Despite the lack of a relationship between baseline mood states and physiological or subjective cue reactivity, there was strong evidence that mood states were associated with the magnitude of drug-related feelings experienced prior to and following cue exposure. In general, subjects who reported higher levels of negative mood also reported higher levels of cocaine craving, high, and withdrawal. As discussed in Section 1, these results are consistent with a number of earlier correlational studies (e.g. Sherman et al., 1986; Weddington et al., 1990; Satel et al., 1991; Kowatch et al., 1992; Tunis et al., 1994; Covi et al., 1995).

These correlations between drug states and negative mood could be interpreted in several ways. First, the correlations could indicate that subjects in treatment feel badly when experiencing forbidden drug-related feelings. In other words, craving, high, and withdrawalcould have been the cause of the negative emotions 
reported on the POMS. Second, negative moods might be a cause of drug craving and associated feelings of high and withdrawal (see Baker et al., 1987). Earlier work (Sherman et al., 1989; Litt et al., 1990; Tiffany and Drobes, 1990; Childress et al., 1994; Maude-Griffin and Tiffany, 1996; Cooney et al., 1997) has demonstrated that induced negative moods can increase craving for cigarettes, opiates, and alcohol. In a similar way, negative moods reported by subjects in the current study might serve as cues which produce craving and other drug states. Finally, the negative mood states experienced by patients might reflect the same emotional states being labeled as craving, withdrawal, and high. Patients may be less capable of discriminating different internal states than we think and may apply the whole range of available mood labels to a single set of feelings. Because the present study was not designed to experimentally manipulate mood states, these alternatives cannot be disentangled.

If we assume that mood states are the cause of the associated drug states experienced prior to the session, then craving caused by drug cues appears to be an independent source of drug craving. Although moods were well-correlated with craving experienced both before and after the session, they were not significantly associated with the magnitude of craving change experienced during cue presentations. Therefore, negative mood states and drug cues could be thought of as separate sources of craving whose effects summate to determine an individual's overall level of drug desire (see Cooney et al., 1997). In that case, future behavioral treatments could be aimed at reducing craving caused both by drug-related cues and negative mood states. Furthermore, putative anticraving medications could be examined for their effects on craving resulting from each source. However, such conclusions await the results of studies employing experimental manipulations of mood states with cocaine abuse patients.

In sum, the present study demonstrates that reactions to cocaine cues in the laboratory are largely independent of between-subject variations in mood state and recent cocaine use. Although previous studies have shown that these variables can influence drug craving, the present results provided no evidence for such an association with craving responses to laboratory cocaine cues. These findings support one of the basic assumptions behind the use of cue reactivity assessments as measures of treatment progress, namely that such sessions reduce within-group variability in craving reports. By reducing at least two uncontrolled sources of variance in craving measurements, cue reactivity sessions increase the power of treatment studies to detect between-group differences in craving.

\section{Acknowledgements}

This work was supported by NIDA Project no. DA03008, NIDA Center Grant P50-DA09252-02, the U.S. Department of Veteran's Affairs and Janssen Pharmaceuticals.

\section{References}

Alim, T.N., Rosse, R.B., Vocci, F.J., Deutsch, S.I., 1994. Open-label, dose run-up study of diethylpropion in initial cocaine abstinence. Clin. Neuropharmacol. 17, 175-187.

Alim, T.N., Rosse, R.B., Vocci, F.J., Lindquist, T., Deutsch, S.I., 1995. Diethylpropion pharmacotherapeutic adjuvant therapy for inpatient treatment of cocaine dependence: a test of the cocaineagonist hypothesis. Clin. Neuropharmacol. 18, 83-195.

Baker, T.B., Morse, E., Sherman, J.E., 1987. The motivation to use drugs: a psychological analysis of urges. In: Rivers, C. (Ed.), The Nebraska Symposium on Motivation, Alcohol Use and Abuse. University of Nebraska Press, Lincoln, NE, pp. 257-323.

Berger, S.P., Hall, S., Mickalian, J.D., Reid, M.S., Crawford, C.A., Delucchi, K., Carr, K., Hall, S., 1996. Haloperidol antagonism of cue-elicited craving. Lancet 347, 504-508.

Burke, W.M., Ravi, N.V., Dhopesh, V., Vandegrift, B., Maany, I., 1990. Prolonged presence of cocaine metabolite in urine after compulsive cocaine use. J. Clin. Psychiatry 51, 145-148.

Childress, A.R., Ehrman, R., McLellan, A.T., MacRae, J., Natale, M., O’Brien, C.P., 1994. Can induced moods trigger drug-related responses in opiate abuse patients? J. Sub. Abuse Treat. 11, $17-23$.

Childress, A.R., Ehrman, R.N., McLellan, A.T., O'Brien, C.P., 1988. Conditioned craving and arousal in cocaine addiction: A preliminary report. NIDA. Res. Monogr. 81, 74-80.

Cooney, N.L., Litt, M.D., Morse, P.A., Bauer, L.O., Gaupp, L., 1997. Alcohol cue reactivity, negative-mood reactivity, and relapse in treated alcoholic men. J. Abnorm. Psychol. 106, 243-250.

Covi, L., Hess, J.M., Kreiter, N.A., Haertzen, C.A., 1995. Effects of combined fluoxetine and counseling in the outpatient treatment of cocaine abusers. Am. J. Drug Alcohol Abuse 21, 327-344.

Drobes, D.J., Meier, E.A., Tiffany, S.T., 1994. Assessment of the effects of urges and negative affect on smokers' coping skills. Behav. Res. Ther. 32, 165-174.

Droungas, A., Ehrman, R.N., Childress, A.R., O'Brien, C.P., 1995. Effect of smoking cues and cigarette availability on craving and smoking behavior. Addict. Behav. 20, 657-673.

Ehrman, R.N., Robbins, S.J., Childress, A.R., O’Brien, C.P., 1992. Conditioned responses to cocaine-related stimuli in cocaine abuse patients. Psychopharmacology 107, 523-529.

Ehrman, R.N., Robbins, S.J., Cornish, J.W., Childress, A.R., O'Brien, C.P., 1996. Failure of ritanserin to block cocaine cue reactivity in humans. Drug Alcohol Depend. 42, 167-174.

Ehrman, R.N., Robbins, S.J., Childress, A.R., Goehl, L., Hole, A.V., O'Brien, C.P., 1998. Laboratory exposure to cocaine cues does not increase cocaine use by outpatient subjects. J. Subst. Abuse Treat. $15,1-5$.

Elash, C.A., Tiffany, S.T., Vrana, S.R., 1995. Manipulation of smoking urges and affect through a brief-imagery procedure: self-report, psychophysiological, and startle probe responses. Exp. Clin. Psychopharmacol. 3, 156-162.

Fischman, M.W., Schuster, C.R., Hatano, Y., 1983a. A comparison of the subjective and cardiovascular effects of cocaine and lidocaine in humans. Pharmacol. Biochem. Behav. 18, 123-127.

Fischman, M.W., Schuster, C.R., Rajfer, S., 1983b. A comparison of the subjective and cardiovascular effects of cocaine and procaine in humans. Pharmacol. Biochem. Behav. 18, 711-716. 
Fischman, M.W., Schuster, C.R., Javaid, J., Hatano, Y., Davis, J., 1985. Acute tolerance development to the cardiovascular and subjective effects of cocaine. J. Pharmacol. Exp. Ther. 235, 677682.

Fischman, M.W., Foltin, R.W., Nestadt, G., Pearlson, G.D., 1990. Effects of desipramine maintenance on cocaine self-administration by humans. J. Pharmacol. Exp. Ther. 267, 760-770.

Foltin, R.W., Fischman, M.W., 1997. Residual effects of repeated cocaine smoking in humans. Drug Alcohol Depend. 47, 117-124.

Greeley, J., Swift, W., Heather, N., 1992. Depressed affect as a predictor of increased desire for alcohol in current drinkers of alcohol. Br. J. Addict. 87, 1005-1012.

Hawks, R.L., Chiang, C.N., 1986. Examples of specific drug assays. NIDA Res. Monogr. 73, 84-112.

Jaffe, J.H., Cascella, N.G., Kumor, K.M., Sherer, M.A., 1989. Cocaine-induced cocaine craving. Psychopharmacology 97, 59-64.

Kowatch, R.A., Schnoll, S.S., Knisely, J.S., Green, D., Elswick, R.K., 1992. Electroencephalographic sleep and mood during cocaine withdrawal. J. Addict. Dis. 11, 21-45.

Kranzler, H.R., Bauer, L.O., 1992. Bromocriptine and cocaine cue reactivity in cocaine-dependent patients. Br. J. Addict. 87, 15371548.

Litt, M.D., Cooney, N.L., Kadden, R.M., Gaupp, L., 1990. Reactivity to alcohol cues and induced moods in alcoholics. Addict. Behav. 15, 137-146.

Loftus, E.F., 1975. Leading questions and the eyewitness report. Cognit. Psychol. 7, 560-572.

Maude-Griffin, P.M., Tiffany, S.T., 1996. Production of smoking urges through imagery: the impact of affect and smoking abstinence. Exp. Clin. Psychopharmacol. 4, 198-208.

McLellan, A.T., Luborsky, L., Woody, G.E., O’Brien, C.P., 1980. An improved diagnostic evaluation instrument for substance abuse patients: The Addiction Severity Index. J. Nerv. Ment. Disord. $168,26-33$.

McNair, D., Lorr, M., Droppleman, L., 1971. Manual: Profile of Mood States. Educational and Industrial Testing Service, San Diego.

Reid, M.S., Mickalian, J.D., Delucchi, K.L., Hall, S.M., Berger, S.P., 1998. An acute dose of nicotine enhances cue-induced cocaine craving. Drug Alcohol Depend. 49, 95-104.

Rhoades, H.M., Grabowski, J., Elk, R., Cowan, K., 1993. Factor stationarity and invariance of the POMS in cocaine patients. Psychopharmacol. Bull. 29, 263-267.

Robbins, S.J., Ehrman, R.N., 1998. Cocaine use is associated with increased craving in outpatient cocaine abusers. Exp. Clin. Psychopharmacology. 6, 217-224.

Robbins, S.J., Ehrman, R.N., Childress, A.R., O’Brien, C.P., 1992. Using cue reactivity to screen medications for cocaine abuse: a test of amantadine hydrochloride. Addict. Behav. 17, 491-499.
Robbins, S.J., Ehrman, R.N., Childress, A.R., O’Brien, C.P., 1997. Relationships among physiological and self-report responses produced by cocaine-related cues. Addict. Behav. 22, 157-167.

Robbins, S.J., Ehrman, R.N., Childress, A.R., O’Brien, C.P., 1999. Comparing levels of cocaine cue reactivity in male and female outpatients. Drug Alcohol Depend. 53, 223-230.

Rosse, R.B., Alim, T.N., Fay-McCarthy, M., Collins, J.P., Vocci, F.J., Lindquist, T., Jentgen, C., Hess, A.L., Deutsch, S.I., 1994. Nimodipine pharmacotherapeutic adjuvant therapy for inpatient treatment of cocaine dependence. Clin. Neuropharmacol. 17, $348-358$.

Satel, S.L., Price, L.H., Palumbo, J.M., McDougle, C.J., Krystal, J.H., Gawin, F., Charney, D.S., Heninger, G.R., Kleber, H.D., 1991. Clinical phenomenology and neurobiology of cocaine abstinence: a prospective inpatient study. Am. J. Psychiatry 148, $1712-1716$.

Satel, S.L., Krystal, J.H., Delgado, P.L., Kosten, T.R., Charney, D.S., 1995. Tryptophan depletion and attenuation of cue-induced craving for cocaine. Am. J. Psychiatry 152, 778-783.

Shadel, W.G., Niaura, R., Abrams, D.B., Goldstein, M.G., Rohsenow, D.J., Sirota, A.D., Monti, P.M., 1998. Scripted imagery manipulations and smoking cue reactivity in a clinical sample of self-quitters. Exp. Clin. Psychopharmacol. 6, 179-186.

Sherman, J.E., Morse, E., Baker, T.B., 1986. Urges/craving to smoke: preliminary results from withdrawing and continuing smokers. Adv. Behav. Res. Ther. 8, 253-269.

Sherman, J.E., Zinser, M.C., Sideroff, S.I., Baker, T.B., 1989. Subjective dimensions of heroin urges: influence of heroin-related and affectively negative stimuli. Addict. Behav. 14, 611-623.

Tiffany, S.T., Drobes, D.J., 1990. Imagery and smoking urges: the manipulation of affective content. Addict. Behav. 15, 531-539.

Tiffany, S.T., Singleton, E., Haertzen, C.A., Henningfield, J.E., 1993. The development of a cocaine craving questionnaire. Drug Alcohol Depend. 34, 19-28.

Tunis, S.L., Delucchi, K.L., Hall, S.M., 1994. Assessing thoughts about cocaine and their relationship to short-term treatment outcome. Exp. Clin. Psychopharmacol. 2, 184-193.

Tversky, A., Kahneman, D., 1973. Availability: a heuristic for judging frequency and probability. Cognit. Psychol. 5, 207-232.

Tversky, A., Kahneman, D., 1974. Judgment under uncertainty: heuristics and biases. Science 125, 1124-1131.

van Gorp, W.G., Wilkins, J.N., Hinkin, C.H., Moore, L.H., Hull, J., Horner, M.D., Plotkin, D., 1999. Declarative and procedural memory functioning in abstinent cocaine abusers. Arch. Gen. Psychiatry 56, 85-89.

Weddington, W.W., Brown, B.S., Haertzen, C.A., Cone, E.J., Dax, E.M., Herning, R.J., Michaelson, B.S., 1990. Changes in mood, craving, and sleep during short-term abstinence reported by male cocaine addicts. Arch. Gen. Psychiatry 47, 861-868. 